\title{
Modeling, Design and Experimentation of Wearable RFID Sensor Tag
}

\author{
Cecilia Occhiuzzi, Stefano Cippitelli, and Gaetano Marrocco
}

\begin{abstract}
Design of effective wearable tags for UHF RFID applications involving persons is still an open challenge due to the strong interaction of the antenna with the human body which is responsible of impedance detuning and efficiency degradation. A new tag geometry combining folded conductors and tuning slots is here discussed through numerical analysis and extensive experimentation also including the integration of a passive motion detector. The achieved designs, having size comparable with a credit card, may be applied to any part of the body. The measured performance indicates a possible application of these body-worn tags for the continuous tracking of human movements in a conventional room.
\end{abstract}

Index Terms - Antennas, biomedical applications of electromagnetic radiation, biomedical telemetry, transponders.

\section{INTRODUCTION}

$\mathbf{T}$ HE possibility to monitor and identify people by means of low-power and low-cost technology is nowadays one of the most interesting and promising features of radio frequency identification (RFID) techniques. Thanks to the advances in low-power electronics, it is now feasible to envisage sophisticated RFID-like devices integrating sensing and signal processing ability [1] able to provide real-time biomonitoring (temperature, blood pressure, heartbeat, glucose content, human behavior) and location of people within hospitals or domestic environment [2]-[5].

The UHF (860-960 MHz) standard is particularly attractive in passive low-cost applications due to the permitted high datarate and large reading distances potentially comparable with the size of typical indoor environments.

The requirements of wearable antennas are small dimensions and lightweight as well as high immunity to the human body interaction which may otherwise sensibly change the radiation diagram and degrade the antenna efficiency. Some of these issues are also common to the design of tags for metal objects whose presence strongly affects the radiation diagrams of the attached antenna and prevents the use of dipole like layouts.

In active and semi-active architectures, as in the case of bodycentric communication systems [6], the overall radiation performance is enhanced by additional battery-assisted electronics. In

Manuscript received July 24, 2009; revised November 23, 2009; accepted February 01, 2010. Date of publication May 18, 2010; date of current version August 05, 2010.

C. Occhiuzzi and G. Marrocco are with the DISP, University of Roma “Tor Vergata," 00133 Roma, Italy (e-mail: occhiuzzi@disp.uniroma2.it; marrocco@ disp.uniroma2.it).

S. Cippitelli was with the DISP, University of Roma "Tor Vergata," 00133 Roma, Italy. He is now with SIA, 10146 Torino, Italy.

Digital Object Identifier 10.1109/TAP.2010.2050435 case of passive tags instead, where the energy to produce the response comes from a remote query unit, the antenna design is much more challenging.

Several solutions have been recently investigated for the design of passive tags over metals, mainly based on the use of high permittivity slabs and of metallic shields, integrated in the antennas as ground planes. Typical antennas are the patch-like family comprising PIFA and IFA layouts, [7] (maximum gain up to $6 \mathrm{~dB}$ using the parasistic constructive effect of the surronding objects), [8] (gain max: $-2 \mathrm{~dB}$ ) [9] (gain max: $-6.4 \mathrm{~dB}$ ).

The design of wearable passive UHF tags has up to now received much less attention. In a previous paper [10], the authors considered a family of slot antennas over a suspended patch, partly decoupled from the body by a silicone slab. The study was mostly oriented to define tuning mechanisms for the required conjugate impedance matching to a great variety of microchip impedances and to understand the dependence of the antennas's bandwidth on the body placement. The antenna layout was intended to host additional electronics and contacting or non-contacting sensors. The maximum size of these antennas was of the order of 4-6 cm and the typical gain was rather poor (gain max: $-7 \mathrm{~dB}$ ) due to the bidirectional radiation of the slot. The expected activation ranges were therefore modest, even if it was demonstrated that the gain may be improved by enlarging the overall size. In [11] the use of automatically optimized slot-line transformers was further investigated for miniaturization and multi-band purposes.

The rich study in [12] (and herein included references) considers some solutions partly decoupled from the body such as multi-folded dipole antennas over a shielding plate and regular patch and PIFA configurations. These devices are specifically designed for wearable applications and experimentally evaluated for what concerns the monitoring of runners in open areas and of personnel inside buildings. Some interesting effects are characterized, such as the influence of the tilt of the transmitting and received antennas and the mutual shadowing among people in the same area. The various antennas are not intended to host sensors but only to identify the person. The dominant size in all the cases was around $15 \mathrm{~cm}$ and the measured on-body gain ranges between $0 \mathrm{~dB}$ and $5 \mathrm{~dB}$ in the largest configurations.

Very recently, new magnetic materials have been considered as a shielding plate for an RFID tag [13]. The innovative ferritesilicone $(\mathrm{BaCo})$ composite promises to achieve very low-profile miniaturized and flexible structures potentially useful for wearable applications. The measured maximum gain in air is of the order of $-7 \mathrm{~dB} \div-4 \mathrm{~dB}$.

This contribution proposes a planar layout which combines the tuning agility of the shaped-slot based tags and the decoupling from the body achieved by grounded antennas. The basic 


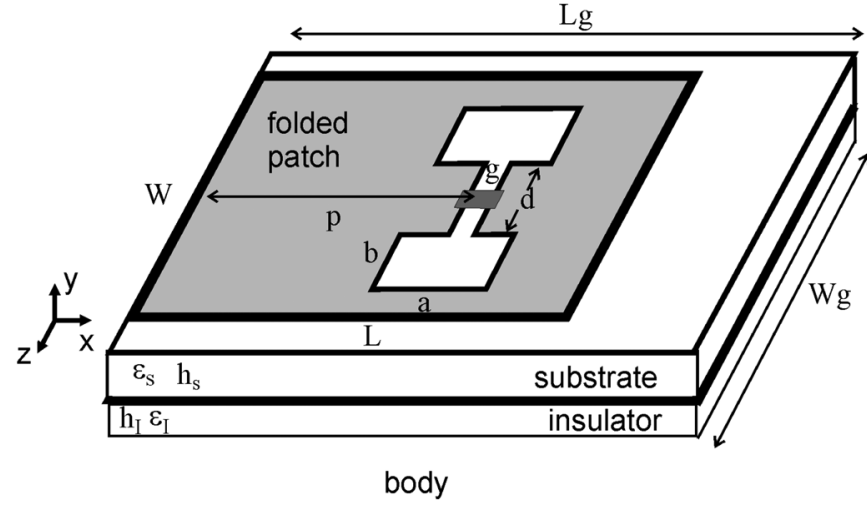

Fig. 1. Layout of the proposed tag family. The $\mathrm{H}$-slot acts as tuning impedance. The sensors may be allocated over the top conductor.

configuration comprises a folded patch sourced by an embedded $\mathrm{H}$-slot whose main features are: on-body gain higher than previous examples in [10] and comparable with that of tags over metal, approximately constant radiation performances regardless of the different body positions, reduced sizes and the predisposition to host passive sensors. A general design procedure is here described to apply the proposed antenna configuration to RFID microchips of given input impedance by the help of an equivalent circuit model (Section II) useful to better understand the electromagnetic role of the antenna's geometrical parameters and to provide a starting guess in the final tag design. The real performances of the tags are then evaluated (Section III) by means of an articulated experimental campaign comprising the input impedance measurement of some prototypes and the read-region characterization when the antenna plus the RFID microchip is attached onto the human body. Finally, the paper describes (Section IV) how the tag design procedure may also account for the electrical features of the sensor in the conjugate impedance matching with reference to the integration of a simple motion sensor. The performance of the resulting integrated antenna is in conclusion analyzed in the detection of typical body movements, in comparison with more accurate accelerometric data.

\section{Antenna Layout AND Design Procedures}

A rectangular plate is folded (Fig. 1) around a dielectric slab of height $h_{S}$ and the longest face is placed over the body through an optional dielectric insulator slab of thickness $h_{I}$. Unlike the shunt-fed conventional PIFA, this geometry can be viewed as a series-fed "L"-patch. An optional strategy to further improve the decoupling with the body, could be the design of a lower plate slightly wider than the upper one $\left(W_{g}>W\right.$ and $\left.L_{g}>L\right)$. The RFID microchip will be attached in the middle of the slot's central gap.

The radiation (Fig. 2) is produced mostly by the slot and the patch's open edge. Assuming that the thickness $h_{S}$ of the inner dielectric is small compared with the wavelength, the radiation from the folding may be considered negligible and the gain and matching features of the antenna are mainly related to the slot and to the transmission line truncation. The polarization

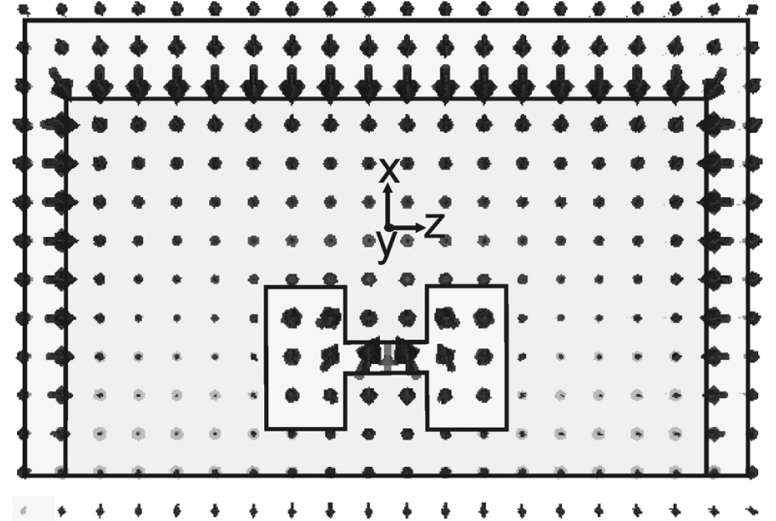

Fig. 2. Near field distribution of the proposed wearable antenna. The radiation is maximum in correspondence of the central slot and the open edge (in opposite phase) and minimum close to the folding. As for conventional patches the fields along the external vertical sides ( $x$ axis in the figure) are in opposite phase, thus do not greatly contribute to the radiation.

is linear, parallel to the antenna main-direction ( $x$ axis in the figure).

As for conventional patches, the increase in the horizontal size $W$ produces a gain enhancement. Depending on the position of the tag on the body, and on the available space, it is possible to increase that dimension in order to achieve better radiation performance. The length $L$ of the patch is chosen approximately equal to $\lambda / 4$, where $\lambda$ is the effective wavelength in the dielectric substrate. While the size of the slot's central gap is mainly fixed by the microchip packaging and by the eventual sensing electronics, different shape-factors and positions may be instead considered for the matching slot.

The maximization of the read distance requires the antenna impedance $Z_{A}$ to match the conjugate microchip impedance $Z_{\text {chip }}^{*}$. To understand the role of the many geometrical variables on the antenna impedance and to achieve a starting guess for the design, an equivalent circuit and a parametric study are here presented.

\section{A. Circuit Model}

Under the hypothesis that the antenna's lower plate is considered as an ideal infinite ground plane the input impedance of the wearable antenna can be predicted by the equivalent circuit in Fig. 3. The above assumption is reasonable if the lower plate is a little larger than the upper antenna face hosting the microchip transponder. It is worth anticipating that both simulative and experimental considerations, to be presented later on, will demonstrate that the antenna's performance is very little sensitive to the placement on different parts of the body thanks to the previously discussed decoupling mechanisms.

The structure is therefore analyzed as a microstrip transmission line truncated by a non-ideal open circuit at the first termination, by a short circuit at the other one and loaded in series by a complex-impedance element: the H-slot. A transformer's turn ratio $n_{p}$ accounts for the coupling of the H-slot to the rectangular plate.

The non ideal open circuit produces fringing field, as in conventional patch antennas and can be accounted for by an open- 


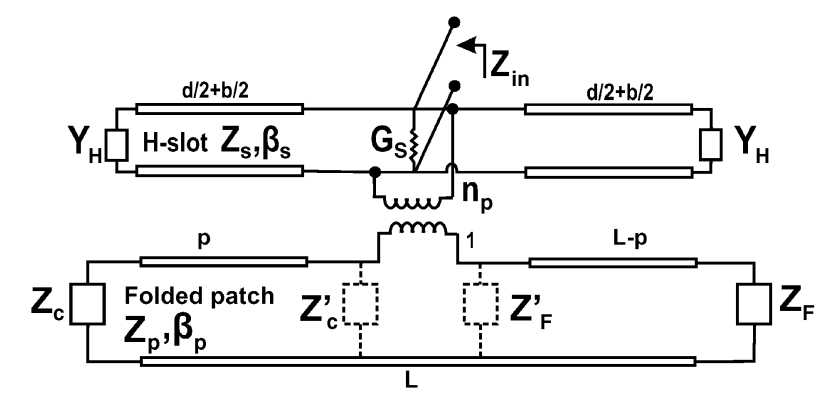

Fig. 3. Equivalent transmission line model of folded patch loaded by the H-slot. Each part of the antenna is modeled as an equivalent impedance/admittance: $Z_{c}$ for the short circuit, $Z_{F}$ for the non ideal open circuit, $Y_{H}$ and $G_{S}$ for the slot, coupled by the transformer's turn ratio $n_{p}$.

ended slot with equivalent parallel admittance $Y_{F}=G_{F}+j B_{F}$ [14] given by

$$
\begin{aligned}
G_{F} & =\frac{W}{120 \lambda_{0}}\left[1-\frac{1}{24}\left(k_{0} \cdot h_{s}\right)^{2}\right] \\
B_{F} & =\frac{W}{120 \lambda_{0}}\left[1-0.636 \ln \left(k_{0} \cdot h_{s}\right)\right] .
\end{aligned}
$$

The other short-circuit truncation of the antenna can be roughly modeled as an inductance [15]

$$
L c=h_{s} \cdot 0.2\left[\log \left(\frac{2 h_{s}}{W+t}\right)+0.2235\left(\frac{W+t}{h_{s}}\right)+0.5\right]
$$

where $t$ is the thickness of the conductive sheet.

The H-slot could be viewed as the combination of three portions of slot-lines. The horizontal $(d+2(b / 2)) \times g$ slot is mainly associated with the coupling and the radiation through a conductance [14]

$$
G_{S}=\frac{d}{120 \lambda_{0}}\left[1-\frac{1}{24}\left(k_{0} \cdot g\right)^{2}\right] .
$$

The two identical vertical longitudinal short-circuit slot-lines of width $b$ and length $a$, as described in [16], host phase-reversal aperture fields, and hence they mainly store reactive energy. The effect of each vertical $b \times a$ slot is accounted for by the series admittance $Y_{H}$ of two short-circuit stubs of length $a / 2$, e.g.

$$
Y_{H}=-\frac{1}{2} j Y_{S} \cot \left(\beta_{s} a / 2\right)
$$

where $Y_{S}$ and $\beta_{S}$ are the characteristic impedance and the wave number of the slot-line with width $a$ calculated as in [17].

Denoting with $Y_{c}^{\prime}$ and $Y_{F}^{\prime}$ the admittance of the microstrip's shorted- and open-ended termination, after transfer up to the microchip connection, and $Y_{H}^{\prime}$ the vertical-slot admittance $\left(Y_{H}\right)$ again transferred at the center of the slot, the total input impedance of the antenna is finally given by

$$
Z_{i n} \frac{n_{p}^{2}\left(Z_{c}^{\prime}+Z_{F}^{\prime}\right)}{1+n_{p}^{2}\left(Y_{H}^{\prime}+G_{S}\right)\left(Z_{c}^{\prime}+Z_{F}^{\prime}\right)}
$$

with $Z_{c}^{\prime}=\left(Y_{c}^{\prime}\right)^{-1}, Z_{F}^{\prime}=\left(Y_{F}^{\prime}\right)^{-1}$. The transformer's turn ratio $n_{p}$ is related to various antenna's parameters such as the slot size and its position along the upper patch. $n_{p}$ is roughly equal to the fraction of the current intercepted by the aperture to the total antenna current and can be calculated numerically, for instance

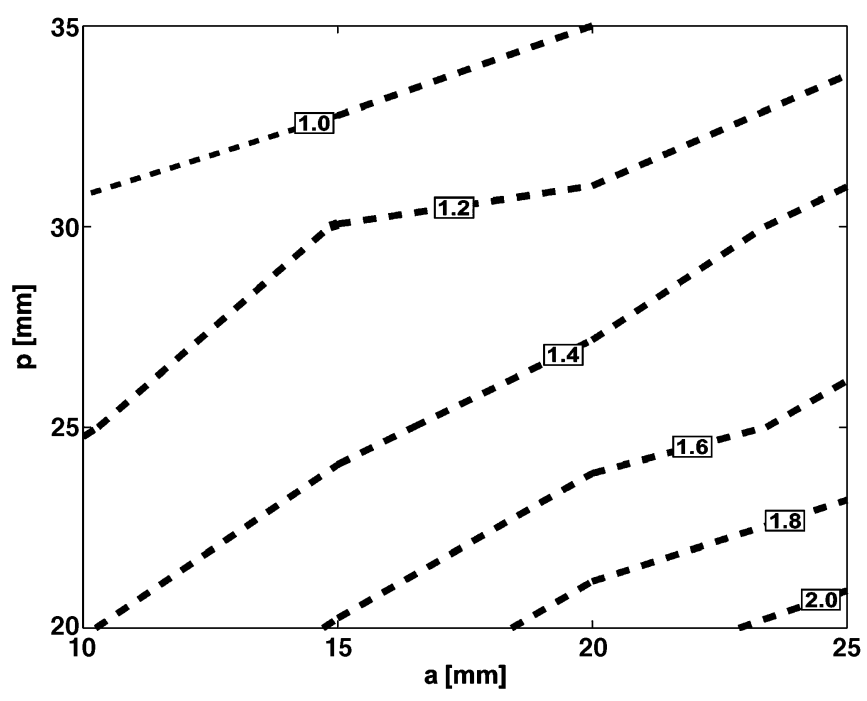

Fig. 4. Variation of the transformer's turn ratio $n_{p}$ of the circuit model with respect to the slot dimension $a$ and its position $p$, having fixed (size in [mm]) $L=46.5, W=80, b=3, d=10, g=2$.

as described in [18], or by means of best fitting of the numerically computed input impedance to the circuital expression in (5). Just for example, Fig. 4 shows the dependence $n_{p}(a, p)$ in the case of PTFE inner dielectric $\left(\varepsilon_{1}=2.08, \tan \delta=4 \cdot 10^{-4}\right)$ and having fixed the other sizes deduced by an FDTD-simulation [19] of the whole structure.

As expected, the amount of current intercepted by the H-slot, and thus the $n_{p}$ ratio, increase for large slots; moreover it is maximum when the slot is close to the left folding $(p=20 \mathrm{~mm})$ and minimum in proximity of the metal plate's open-circuit truncation $(p=35 \mathrm{~mm})$. The variation of the turn ratio is well approximated by a bilinear polynomial fitting (with respect to $a$ and $p[\mathrm{~mm}])$

$$
n_{p} \approx 2.184+0.0328 a-0.0530 p .
$$

\section{B. Parametric Analysis}

Fig. 5 and Fig. 6 show the variation of the tag's input impedance versus the position $p$ and versus the shape factor of the matching slot (modified by acting only on the parameter a) when the inner dielectric is again the PTFE with the same thickness as before. The antenna reactance is inductive before the first resonance and hence this configuration is suited to achieve conjugate matching to the capacitive impedance of the microchip. Moreover, the resistance and reactance change in an opposite way with respect to $p$ and $a$, e.g., the antenna impedance increases (the resonance moves to the lower frequencies) as the slot moves closer to the folding ( $p$ reduces) while it reduces (the resonance moves to the higher frequencies) as the $\mathrm{H}$-slot becomes narrower (parameter $a$ reduces). The tag design may therefore concentrate on the optimization of the only $\{a, p\}$ parameters having fixed the remaining ones.

The insets of Fig. 5 and Fig. 6 show the good agreement in the European RFID band of the impedance estimations from circuit model with fullwave FDTD simulation of the planar antenna. The expression in (5) can be therefore used to give a 

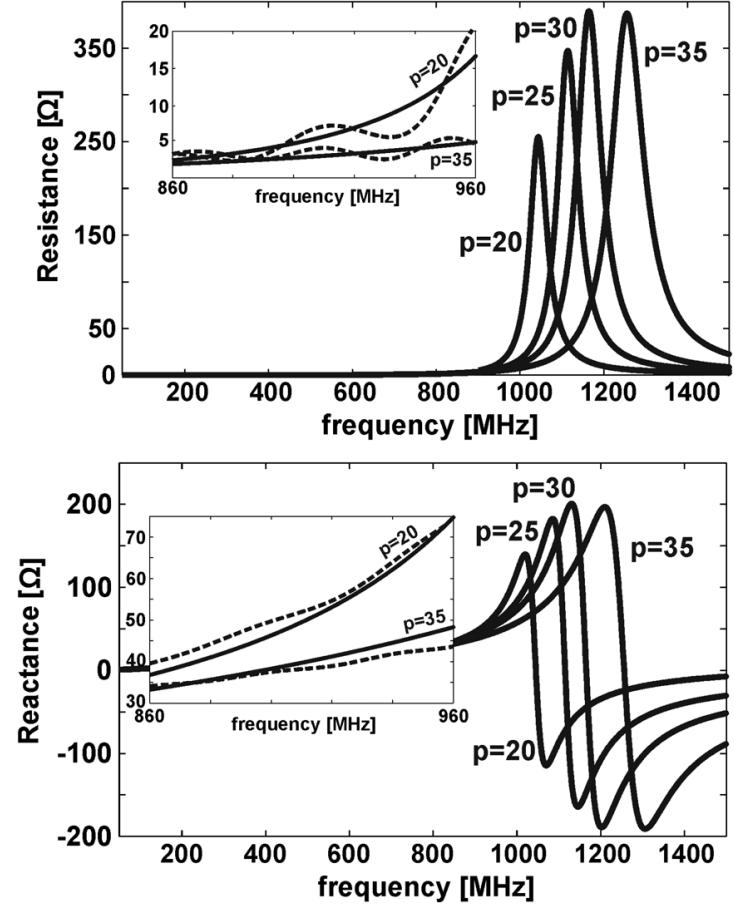

Fig. 5. Parametric exploration of input impedance for various slot positions in $x$ direction (Fig. 1), having fixed (size in [mm]) $L=46.5, W=80, a=20$, $b=3, d=10, g=2$. Continuous lines tag the circuit data while the dashed lines indicate the fullwave results.
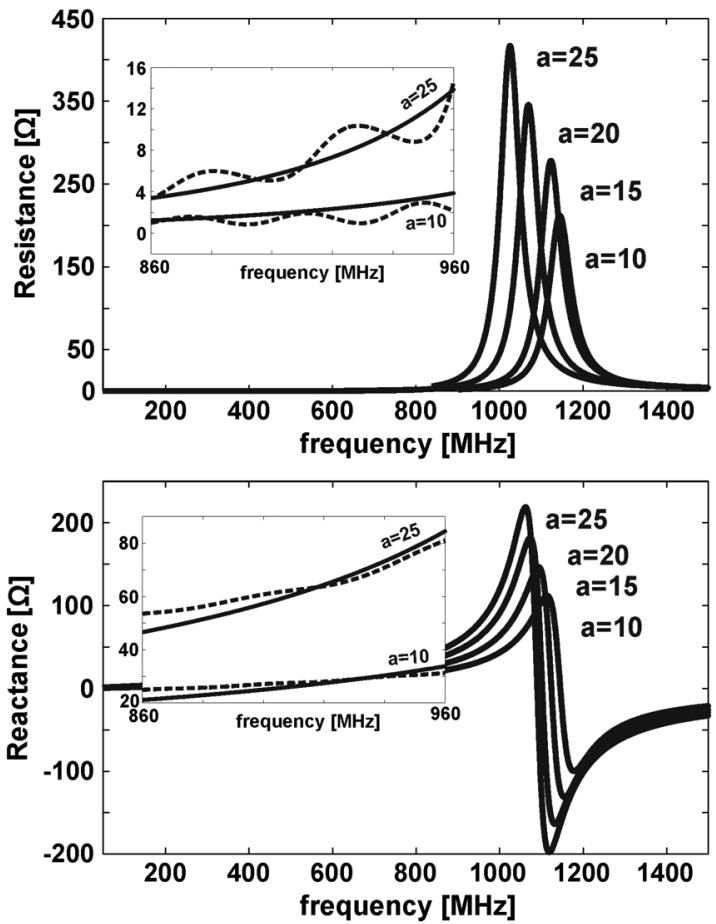

Fig. 6. Parametric exploration of the input impedance for various slot form factors (selected by the parameter $a$ ), having fixed (size in [mm]) $L=46.5$, $W=80, p=25, b=3, d=10, g=2$. Continuous lines tag the circuit data while the dashed lines indicate the fullwave results.

first approximation for slot's sizes and position such to achieve the impedance matching with the microchip, in view of using a fullwave electromagnetic solver to refine the geometrical parameters.
TABLE I

PARAMETERS OF THE SIMPLIFIED LIMB MODEL AT $870 \mathrm{MHz}$

\begin{tabular}{c|ccc}
\hline Layer & $\varepsilon_{r}$ & $\begin{array}{c}\sigma \\
{[\mathrm{S} / \mathrm{m}]}\end{array}$ & $\begin{array}{c}\text { Cross } \\
\text { section }[\mathrm{cm}]\end{array}$ \\
\hline skin + fat & 14.5 & 0.25 & $12 \times 11$ \\
muscle & 55.1 & 0.33 & $10 \times 9$ \\
bone & 20.8 & 0.33 & $4 \times 2$ \\
\hline
\end{tabular}

TABLE II

PARAMETERS OF THE TAG PROTOTYPES IN [mm]

\begin{tabular}{c|cc}
\hline Parameter & TAG-1 & TAG-2 \\
\hline$W_{g}$ & 60 & 90 \\
$W$ & 60 & 80 \\
$L_{g}$ & 49 & 60 \\
$L$ & 49 & 49 \\
$p$ & $L / 2$ & 15 \\
$a$ & 8 & 18 \\
$b$ & 10 & 9 \\
$d$ & 10 & 10 \\
$g$ & 3 & 4 \\
$h_{S}$ & 4 & 4 \\
\hline
\end{tabular}

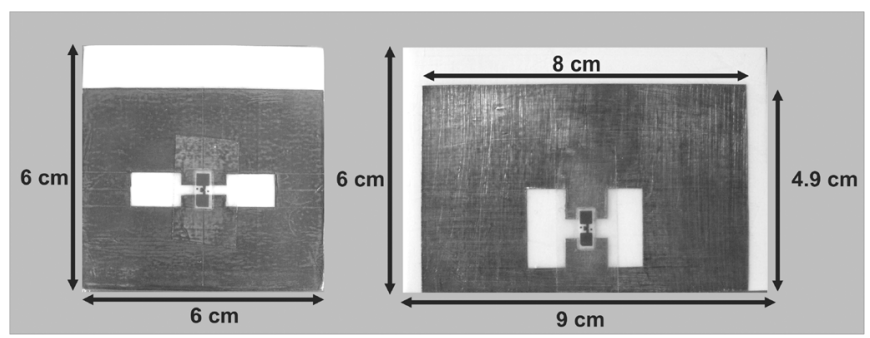

Fig. 7. Fabricated TAG-1 (left) and TAG-2 (right) prototypes of body-worn antenna matched to $Z$ in $=15-j 135 \Omega$ microchip.

\section{Prototypes AND Performances IN REAL CONFIGURATIONS}

Two prototypes of this class of tags have been designed, fabricated and tested in real conditions. The antennas' matching is referred to a low impedance NXP microchip transponder with impedance $Z_{\text {chip }}=15-j 135 \Omega$. The final antenna design has been refined by including into the FDTD simulation also a rough model of human limb consisting of a stratified box of height 40 $\mathrm{cm}$ (parameters in Table I).

The resulting fabricated prototypes, of overall size $6 \times 6 \mathrm{~cm}$ (TAG-1) and $6 \times 9 \mathrm{~cm}$ (TAG-2), (other parameters in Table II) are shown in Fig. 7. The insulating dielectric, contacting the body, is a thin adhesive PVC film.

TAG-2 is expected to have a higher gain in comparison with TAG-1 $\left(G_{2, \max }=0 \mathrm{~dB}\right.$ v.s. $G_{1, \max }=-3 \mathrm{~dB}$, as estimated by FDTD) thanks to the larger $W$, and to the wider ground plane which prevents the antenna radiation to be absorbed into the highly-dissipative human body.

Two different experimental characterizations of the tags' performances are here presented. The antenna design is first checked in chipless modality by the measurement of input impedance $Z_{A}$ used to calculate the power transmission coefficient

$$
\tau=\frac{4 R_{\text {chip }} R_{A}}{\left|Z_{\text {chip }}+Z_{A}\right|^{2}}
$$




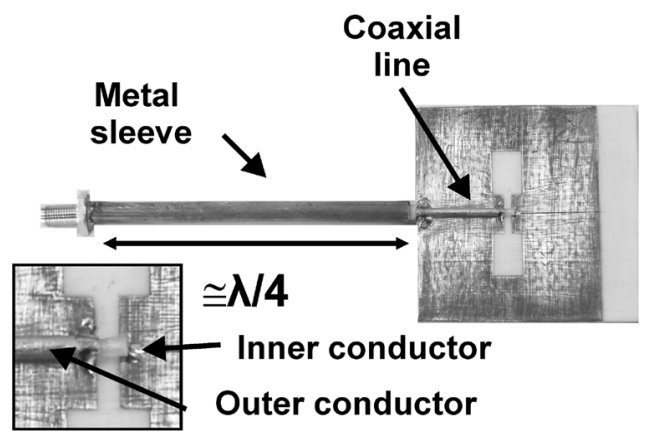

Fig. 8. Antenna plus balun connection. The external conductor of the coaxial line, coming out of the balun, is soldered to the antenna face up to the slot, while the internal conductor is soldered to the other slot's edge.

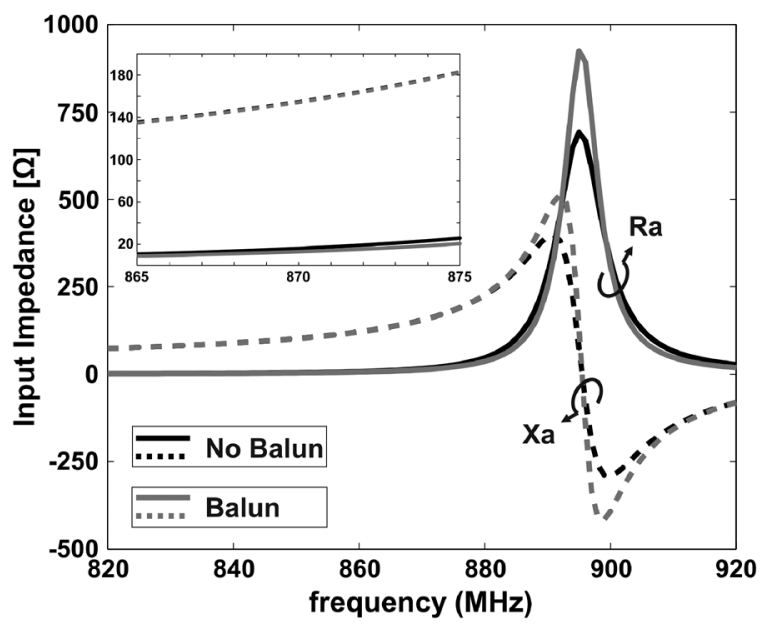

Fig. 9. FDTD simulated input impedance of TAG-1 with and without the presence of the balun.

The RFID link performance is instead fully analyzed having attached the microchip at the antenna port and by estimating the maximum read-distance in controlled conditions, as explained in details later on.

\section{A. Chipless Measurements: Matching Features}

The tags' impedance has been measured by means of a Vector Network Analyzer, VNA (Anritsu MS2024A) probe connected to the slot mid-point through a bazooka balun having the purpose to prevent unbalanced currents from the probe to flow on the outer surface of the coaxial cable [14]. An approximately $\lambda / 4$ metal sleeve, shorted at one termination encapsulates the coaxial probe (Fig. 8). The input impedance measured by the VNA will be hence the tag impedance itself without artifacts. As a proof, Fig. 9, shows a comparison between the simulated tag without cable and balun, and the also simulated impedance in the measurement condition. As visible the input impedance of the tag plus the balun, estimated by FDTD, is practically unchanged, at least in the RFID band, with respect to the standalone antenna.

The antenna has been attached over the leg of a volunteer and the measurement of impedance, after de-embedding of the coaxial connector, gives a power transmission coefficient (at 869 MHz) of the order of $\tau \simeq 0.7$ (TAG-1) and $\tau \simeq 0.85$ (TAG-2)
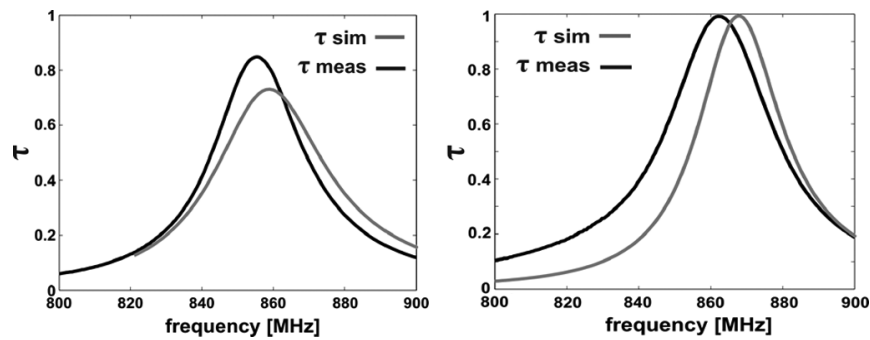

Fig. 10. Simulated and measured power transmission coefficient $\tau$ for the two antennas matched to $Z_{c h i p}=15-j 135$. Left: TAG-1. Right: TAG-2.

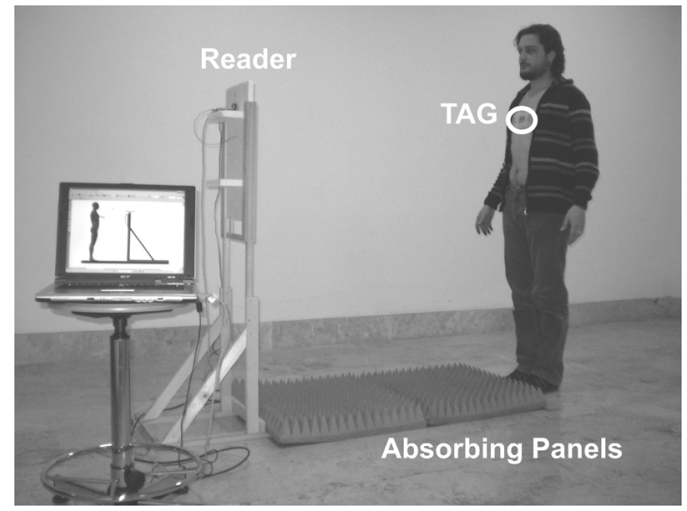

Fig. 11. Measurement setup comprising the short-range reader, the measurement trick and the absorbing panels. The antenna is here placed in the center of the human torso.

(Fig. 10). It is worth mentioning that nearly identical results are obtained when the tags are placed onto different body segments, such as the torso and the arm, as shown in the next paragraph concerning the measurement of the realized gain.

\section{B. Realized Gain}

The realized gain of the tags, e.g. the radiation gain of the antenna reduced by the impedance mismatch, has been indirectly estimated for TAG-1 and TAG-2 by using the set-up in Fig. 11 comprising a short-range, remotely controlled reader CAEN A528, and a quarter-lambda patch (PIFA) with maximum gain $3.3 \mathrm{~dB}$, as reader's antenna. Under the free-space assumption, the power delivered by the reader to the tag, placed at mutual distance $d$, is given by the Friis formula

$$
P_{R \rightarrow T}=\left(\frac{\lambda_{0}}{4 \pi d}\right)^{2} G_{R}(\theta, \phi) G_{T}(\theta, \phi) \tau \eta_{p} P_{i n}
$$

where $G_{R}$ is the gain of the reader antenna, $G_{T}$ is the gain of the tag's antenna, placed on the target. $P_{i n}$ is the power accepted by the antenna of the reader unit. The polarization mismatch $\eta_{p}$ between the reader and the tag is here considered unitary since they have been properly aligned in all the measurements. $G_{T} \tau \equiv \hat{G}_{T}$ is the realized gain of the tag.

Equation (8) has been verified to hold also in a real environment if the measurement set-up is far from the side walls, the distance $d$ is small enough $(d<1 \mathrm{~m})$ and absorbing panels are placed on the ground to reduce multipath. In this case by increasing the reader's power until the tag starts to respond, the collected power at turn-on equals the chip sensitivity, $P_{R \rightarrow T}=$ 


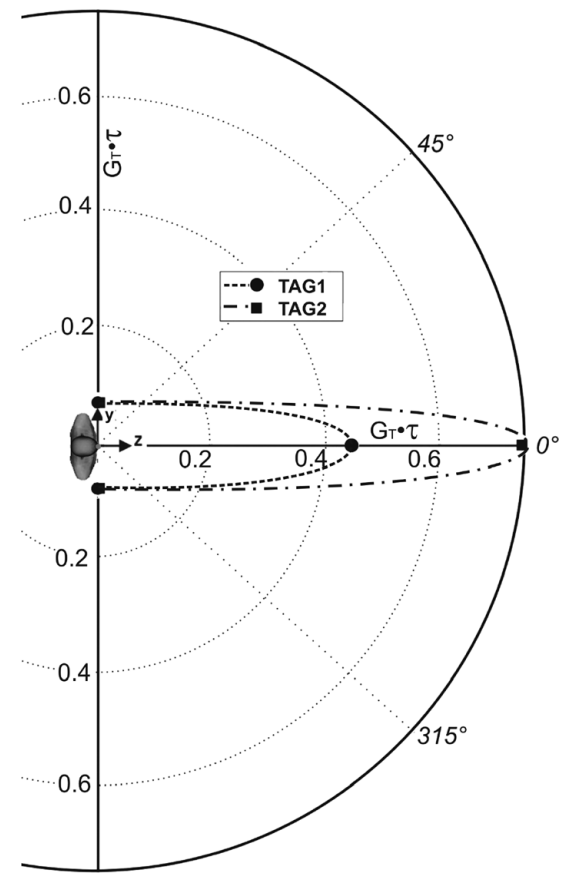

Fig. 12. Measured $\hat{G}_{T}$ for the antenna placed on the torso of the volunteer (top view schematically represented in the origin of the polar graph).

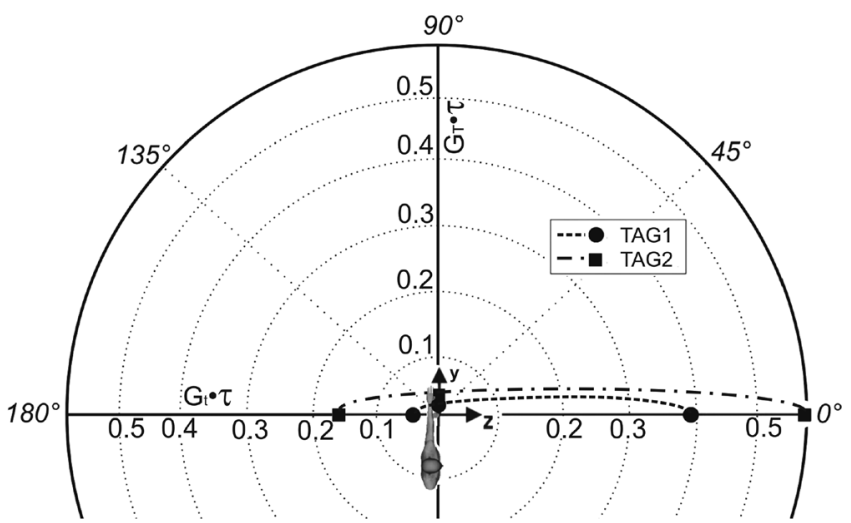

Fig. 13. Measured $\hat{G}_{T}$ for the antenna placed on the left arm of the volunteer (top view schematically represented in the origin of the polar graph).

$P_{c h i p}$, and hence the realized gain can be estimated by inverting (8), when all the other parameters are known.

The measured $\hat{G}_{T}$ is shown in Fig. 12 and Fig. 13 for the tags placed onto two different body regions, the torso and the left arm. The tags are attached onto the body such that the antenna polarization ( $x$ axis in Fig. 1) is parallel to the body's longitudinal axis. $\hat{G}_{T}$ has been evaluated along the two principals directions ( $y$ - and $z$-axis in the figures) by body rotation of $90^{\circ}$, $180^{\circ}$ and $270^{\circ}$. As expected, the realized gain is maximum in front of the antenna while it is minimum in the rear side, due to the human body absorption. However both tags are readable in the back direction when placed on the arms.

The maximum effective gain for the TAG-1 ranges between $-4 \mathrm{~dB}$ and $-3 \mathrm{~dB}$ depending on the body positions, while better performances are achieved by TAG-2, thanks to its larger size, with maximum realized gain ranging between $-2 \mathrm{~dB}$ and -1 $\mathrm{dB}$. These results are in full agreement with the design data.

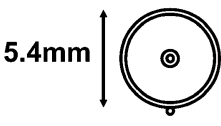

a)

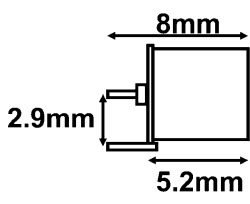

b)

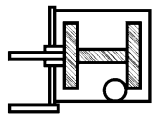

c)
Fig. 14. MS24M motion-vibration sensor design. (a) Bottom view; (b) Side view; (c) Longitudinal section with the inner conductive structure and the switch mass [21].

The radiation performance is hence nearly the same for the two positions, confirming that the antenna is very little sensitive to the body position. It is worth mentioning that the tags still retain similar performance in free space conditions.

The experienced maximum read distance, by using the shortrange reader (emitting not more than $0.5 \mathrm{~W}$ EIRP), and tags' microchip with typical $P_{\text {chip }}=-30 \mathrm{dBm}$, was $1.5 \mathrm{~m}$ for the TAG-1 and $2.1 \mathrm{~m}$ for the TAG-2. However, by using a longrange reader (emitting up to $3.2 \mathrm{~W}$ EIRP) the maximum read distance estimated from (8) could reach $4 \mathrm{~m}$ for the smaller design and $5.5 \mathrm{~m}$ for the larger one.

\section{SENSOR INTEGRATION}

It is here shown how the tag design procedure can be modified to take the presence of a specific sensor into account. As an example, a very simple mechanical motion sensor is considered, and a fully integrated wearable sensor RFID tag is designed, fabricated and hence experimentally evaluated. The detection of the motion, in particular, is of great interest in medical application, to assist the diagnosis of some neurological diseases, involving compulsory arms movements [20], in domestic healthcare, to track the behavior of elderly, but also in logistic and security to control limited-access areas.

\section{A. Omnidirectional Motion Sensor}

The sensor used here is a two terminal omnidirectional switch (Fig. 14) especially designed for the detection of movements and vibrations [21]. When disturbed from its quite condition, it produces fleeting changes of its equivalent impedance state, e.g. open to close or vice versa, and if properly conditioned to the antenna microchip, it may consequently enable or deny the RFID communication. One of the two pins of the steel-gold plated capsule is connected to the external case of the sensor while the other one is isolated from the outer part of the capsule and connected to the inner switching structure (Fig. 14(c)). The switching structure comprises a dumbbell-like conductive element connected to the central pin and a conductive sphere, free to move inside the capsule.

The sensor has two possible states. In state A the internal sphere touches at a same time the inner and the outer conductors of the sensor thus shorting the output pins. In state B, the sphere does not connect the structures and the circuit remains approximately open. State A is stable while state B is instable: at rest the switch is preferably in state A and during the movement it randomly changes between A and B varying its input impedance. 


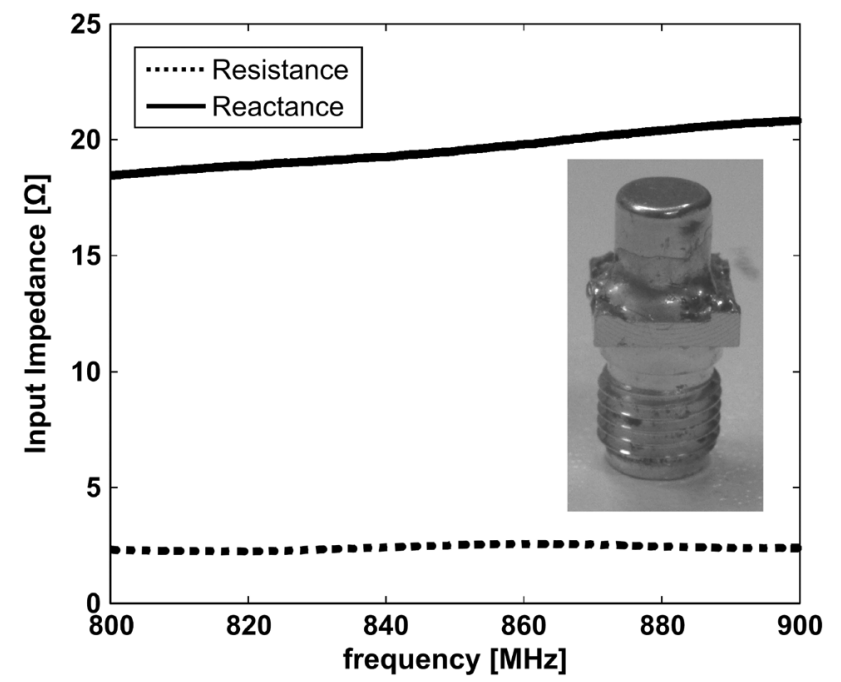

Fig. 15. Measured input Impedance of the motion sensor measured with a VNA probe connected to the switch by means of a modified SMA connector.

The sensor will be connected in series with the microchip and hence the antenna design requires to properly account for the presence of the sensor, e.g., the conjugate matching condition becomes

$$
Z_{A}+Z_{s}^{*}(\text { State A })=Z_{\text {chip }}^{*}
$$

In this choice, the reader will receive the tag ID when the tag is at rest and does not receive anything if the tag is subjected to motion. The basic principle is a form of ID-modulation introduced in [22].

The RF impedance of the switch is not provided by the manufacturer and hence it has been measured with a VNA probe connected to the switch by means of a modified SMA connector (Fig. 15 inset). To avoid the unbalancing effects of the VNA coaxial cable, the capsule has been soldered directly all along the connector flange and its central pin has been inserted in the SMA inner conductor.

At rest (ideally a short circuit), the sensor's measured impedance at $870 \mathrm{MHz}$ is $Z_{s}($ State $\mathrm{A}) \approx 2.5+j 20 \Omega$, therefore showing a practically inductive reactance. The switch's impedance in state B is not easily measurable. During the motion, the sphere randomly moves inside the capsule varying the sensor's impedance without regularity. Basically when the sphere does not touch the sensor walls the resulting impedance is expected to be capacitive with value depending on the instantaneous sphere-wall distance.

\section{B. RFID Motion Sensor Prototype}

A prototype of the wearable Motion Sensor (Fig. 16) comprises a modified version of the TAG-2, with a slightly different slot size in order to achieve the matching condition in (9) having considered the sensor in series to the chip. It is worth noticing that the slot tuning has been accomplished by varying the only slot shape factor (the vertical dimension $a=16 \mathrm{~mm}$ has been changed from $18 \mathrm{~mm}$ to $16 \mathrm{~mm}$ ), leaving unaltered all the other parameters. In order to easily solder the inertial switch, a pack-

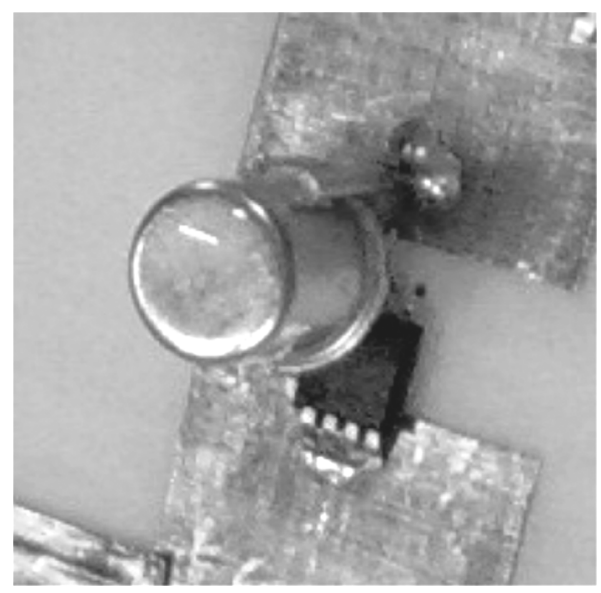

Fig. 16. Particular of the prototype of the TAG-2 Motion Sensor. The inertial switch has been soldered in series to the microchip within the slot central gap.

aged version of the microchip $\left(Z_{\text {chip }}=22-j 162 \Omega\right)$ has been used instead of the strap version.

\section{Experimentation and Results}

The proposed RFID motion sensor has been tested in real conditions in order to verify the effective communication and sensing performances. The movements have been also recorded by 3-axis MEMS motion sensor (LIS302DL [23]), able to measure the accelerations on the three orthogonal axis up to $2 g$ (with $g=9.8 \mathrm{~m} / \mathrm{s}^{2}$ gravitational acceleration) with a sampling rate up to $400 \mathrm{~Hz}$. The MEMS sensor has been placed behind the RFID tag in order to be affected by the same acceleration of the RFID device.

The measurement setup is visible in Fig. 17. Both MEMS sensor and RFID Motion sensor have been placed on the arm and a sixteen-movements sequence has been executed moving the arm randomly. Fig. 18 shows the module of the recorded MEMS sensor vectorial data (a) and the on/off data received at the reader (b), where the bars indicate the state $\mathrm{B}$ (motion). The reader-tag distance and the interrogation power are such that the RFID link may be in principle established for any position of the arm.

A significant correlation is visible between the two motion sensors, in term of number of movements, time and duration. In particular, the RFID Motion Sensor is able to monitor every body event, regardless its standing or magnitude and, when placed onto the chest, it revealed also sensitive to very weak movements such as those produced by deep breath and couch.

\section{CONCLUSIONS}

The analytic model and the detailed experimentations have demonstrated that the proposed family of wearable tags is a good candidate to the monitoring of people in conventional indoor and outdoor area. Thanks to the particular folded geometry, the structure is not much influenced by the detuning and by the absorbing effects produced by the human body. Thanks to the slot, it offers some degree of freedom in the impedance matching, useful to integrate passive sensors.

Further improvements will concern the realization of flexible conformal prototypes based on the textile technology and the in- 


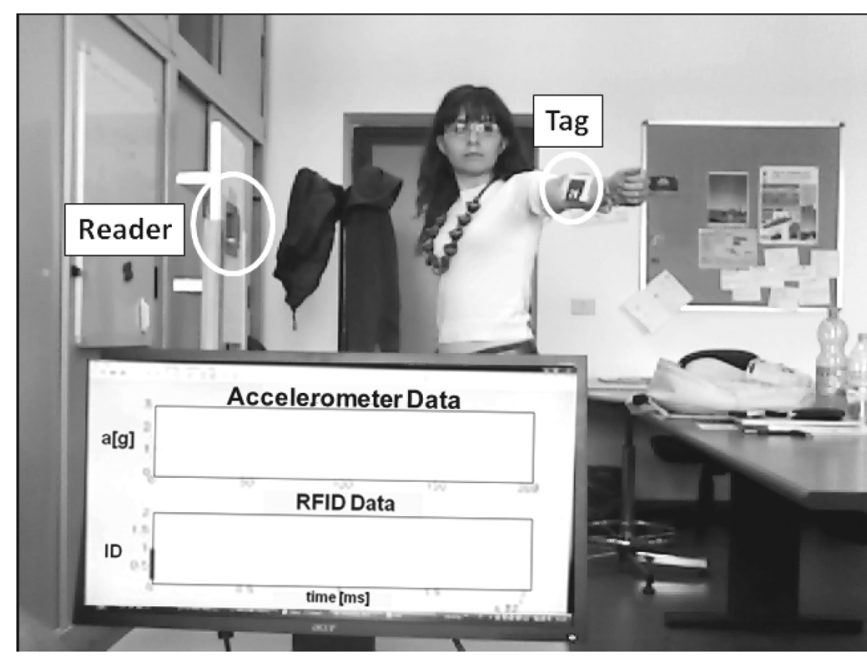

Fig. 17. Measurement setup comprising the short-range reader, the RFID tag and the LIS302DL accelerometer placed beside the tag. Both the RFID motion sensor and the accelerometer are placed on the arm. The MEMS accelerometer data is transmitted via a WIFI module.
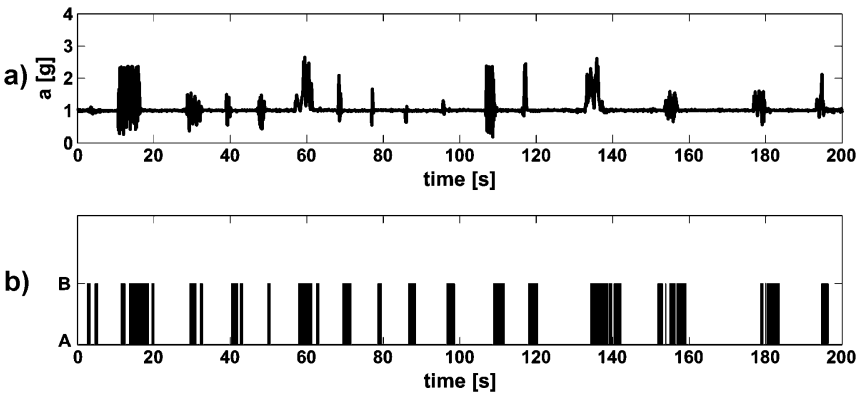

Fig. 18. Comparison of data returned by the LIS302DL accelerometer (a) with the tag response received from the TAG2 Motion Sensor (b): the motion events (state B), for which the microchip does not respond, are indicated by bars.

tegration of a second "control" microchip, whose ID should be received in any condition, revealing the presence and the identity of the tag, leaving to the sensor-conditioned microchip the only duty to communicate the "state" of the tagged object.

\section{ACKNOWLEDGMENT}

The authors wish to thank CAEN for technical support with the reader programming and NXP for providing RFID dies. Special thanks to F. Amato and S. Caizzone for their enthusiastic and valuable support in performing experiments and tests.

\section{REFERENCES}

[1] S. Nambi, S. Nyalamadugu, S. M. Wentworth, and B. A. Chin, "Radio frequency identification sensors," in Proc. 7th World Multiconf. Systemics, Cybernetics and Informatics (SCI 2003), 2003, pp. 386-390.

[2] L. Cheng-Ju, L. Li, C. Shi-Zong, W. C. Chen, H. Chun-Huang, and C. Xin-Mei, "Mobile healthcare service system using RFID," in Proc. IEEE Int. Conf. Networking Sensing and Control, 2004, vol. 2, pp. 1014-1019.

[3] R. S. Sangwan, R. G. Qiu, and D. Jessen, "Using RFID tags for tracking patients, charts and medical equipment within an integrated health delivery network," in Proc. IEEE Int. Conf. Networking Sensing and Control, 2004, pp. 1070-1074.
[4] L. Yang, R. Vyas, A. Rida, J. Pan, and M. M. Tentzeris, “"Wearable RFID-enabled sensor nodes for biomedical application'," presented at the Electronic Components and Technology Conf., Lake Buena Vista, FL, 2008.

[5] J. Park, J. Seol, and Y. Oh, "Design and implementation of an effective mobile healthcare system using mobile and RFID technology," in Proc. 7th Int. Symp. HEALTCOM, 2205, 2005, pp. 263-266.

[6] P. S. Hall and Y. Hao, Antennas and Propagation for Body-Centric Wireless Communications, 1st ed. Norwood, MA: Artech House, 2006.

[7] L. Ukkonen, M. Schaffrath, D. W. Engels, L. Sydänheimo, and M. Kivikoski, "Operability of folded microstrip patch-type tag antenna in the UHF RFID bands within 865-928 MHz," IEEE Antennas Wireless Propag. Lett., vol. 5, pp. 414-417, 2006.

[8] M. Hirvonen, K. Jaakkola, P. Pursula, and J. Saily, "Dual-band platform tolerant antennas for radio-frequency identification," IEEE Trans. Antennas Propag., vol. 54, no. 9, p. 2632, Sep. 2009.

[9] S. L. Chen and K. H. Lin, "A slim RFid tag antenna design for metallic object applications," IEEE Antennas Wireless Propag. Lett., vol. 7, pp. 729-732, 2008.

[10] G. Marrocco, "RFID antennas for the UHF remote monitoring of human subjects," IEEE Trans. Antennas Propag., vol. 55, no. 6, pp. $1862-1680$, June 2007.

[11] C. Calabrese and G. Marrocco, "Meandered-slot antennas for sensorRFID tags," IEEE Antennas Wireless Propag. Lett., vol. 7, pp. 5-8, 2008.

[12] M. Polívka, M. Švanda, and P. Hudec, "UHF RFID of people," in Development and Implementation of RFID Technology. Vienna: I-Tech Education and Publishing, 2009, ch. 4.

[13] L. Yang, L. Martin, D. Staiculescu, C. P. Wong, and M. M. Tentzeris, "Conformal magnetic composite RFID for wearable RF and BIO-monitoring applications," IEEE Trans. Microw. Theory Tech., vol. 56, no. 12, pp. 3223-3230, Dec. 2008.

[14] C. A. Balanis, Antenna Theory: Analysis and Design, 2nd ed. New York: Wiley, 1997

[15] R. Garg, P. Bhartia, I. Bahl, and A. Ittipiboon, Microstrip Antenna Design Handbook. Boston, MA: Artech House, 2001.

[16] M. El Yazidi, M. Himdi, and J. P. Daniel, "Transmission line analysis of nonlinear slot coupled microstrip antenna," Electron. Lett., vol. 28, no. 15 , pp. 1406-1408, 1992.

[17] M. Himdi and J. P. Daniel, "Characteristics of sandwich slot lines in front of parallel metallic strip," Electron. Lett., vol. 21, no. 5, pp. 455-457, 1991

[18] J. P. Kim and W. S. Park, “'Analysis and network modeling of an aperture-coupled microstrip patch antenna'," IEEE Trans. Antennas Propag., vol. 49, no. 6, pp. 849-854, 2001.

[19] G. Marrocco and F. Bardati, "BEST: A finite-difference solver for time electromagnetics," Simul. Practice Theory, no. 7, pp. 279-293, 1999.

[20] W. Hening, "The clinical neurophysiology of the restless legs syndrome and periodic limb movements. Part I: Diagnosis, assessment, and characterization," Clin. Neurophys., vol. 115, pp. 1965-1974, 2004.

[21] MS24M Product Data Sheet Comus Group of Companies [Online]. Available: www.comus-intl.com

[22] M. Philipose, J. R. Smith, B. Jiang, A. Mamishev, S. Roy, and K. Sundara-Rajan, "Battery-free wireless identification and sensing," IEEE Pervasive Comput., vol. 4, no. 1, pp. 37-45, 2005.

[23] "LIS302DL, MEMS Motion Sensor Data Sheet," STMicroelectronics [Online]. Available: www.st.com

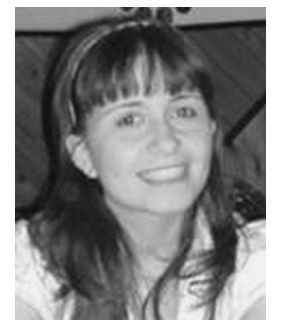

Cecilia Occhiuzzi received the M.Sc. degree in medical engineering from the University of Rome "Tor Vergata" where she is currently working toward the Ph.D. degree.

In 2008, she was at the School of Engineering, University of Warwick, U.K., as a Postgraduate Student. Her research is mainly focused on wireless health monitoring by means of radiofrequency identification (RFID) and UWB techniques. 


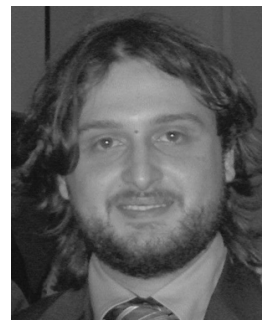

Stefano Cippitelli received the Laurea degree in Telecommunications Engineering from the University of Rome "Tor Vergata," in 2009.

His main scientific interest concerns the design of antenna systems for RFID applications. In spring 2008, he was at Tampere University of Technology, for the advanced course "Design and Characterization of Passive RFID Systems." He is currently employed at SIA, Torino, Italy, working on BTS design.

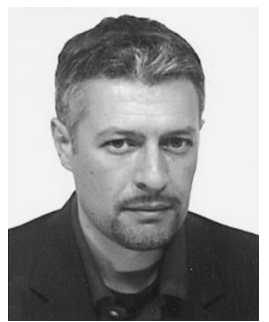

Gaetano Marrocco was born on August 19, 1969, in Teramo, Italy. He received the Laurea degree in electronic engineering and the Ph.D. degree in applied electromagnetics from the University of L'Aquila, Italy, in 1994 and 1998, respectively.

He has been a Researcher at the University of Rome "Tor Vergata" since 1997 where he currently teaches antenna design and bioelectromagnetics. In summer 1994, he was at the University of Illinois at Urbana Champaign, as a Postgraduate Student. In autumn 1999, he was a Visiting Scientist at Imperial College in London. His research is mainly directed to the modelling and design of broadband and ultrawideband antennas and arrays as well as of miniaturized antennas for RFID applications. He has been involved in several space, avionic, naval and vehicular programs of the European Space Agency, NATO, Italian Space Agency, and the Italian Navy. He holds two patents on broadband naval antennas and one patent on sensor RFID systems.

Prof. Marrocco currently serves as an Associate Editor of the IEEE ANTENNAS AND WiRELESS PROPAGATION LETTERS. 